\title{
Weight analysis for various prohibitory sign detection and recognition using deep learning
}

\author{
Christine Dewi ${ }^{1,2} \cdot$ Rung-Ching Chen ${ }^{1} \cdot$ Hui $\mathrm{Yu}^{3}$
}

\begin{abstract}
Traffic sign recognition is meaningful for real-world applications such as self-sufficient driving, traffic surveillance, and driver safety. However, traffic sign recognition is a hard problem because different sizes, illuminations, and noises affect the sign detection and recognition. This work recognizes Taiwan's prohibitory signs using deep learning methods. First, we develop a traffic sign database since there is no such kind of database available in Taiwan. Next, we adopt three different You Only Look Once (Yolo) networks (Yolo A, Yolo B, and Yolo C) and three various Yolo V3 SPP networks (Yolo D, Yolo E, and Yolo F) for prohibitory sign recognition. Finally, we conduct the comparative experiment of Yolo V3 and Yolo V3 SPP with different weights provided by the darknet framework (the best weight, the final weight, and the last weight). Experimental results show that the mean average precision $(m A P)$ observation of all models that the Yolo V3 SPP is better than other models. Yolo D took the optimum average accuracy at 99.0\%, followed by Yolo E and Yolo F 98.9\%. The accuracy of Yolo V3 SPP is growing within the detection time, but it needs more time to identify the sign.

Keywords Weight analysis · Object detection · Traffic sign recognition · Spatial pyramid pooling · Yolo V3
\end{abstract}

\section{Introduction}

Computer vision-based traffic sign detection and recognition have been studied for some purposes, such as Advanced Driver Assistance Systems (ADAS) [34, 35], Auto Driving

Rung-Ching Chen

crching@cyut.edu.tw

1 Department of Information Management, Chaoyang University of Technology, Taichung, Taiwan

2 Faculty of Information Technology, Satya Wacana Christian University, Salatiga, Central Java, Indonesia

3 School of Creative Technologies, The University of Portsmouth, Portsmouth, UK 
Systems (ADS) [27] and traffic surveillance. Detecting and recognizing traffic signs are challenging tasks due to several issues, including rotation, occlusion, color variation, and skewing from camera setup in the environment. Moreover, an image with varying sizes, shapes, and colors may contain multiple signs [43, 46].

The arrival of modern breakthroughs in deep learning [36] has significant state-of-the-art results for recognition tasks and object detection [14, 25, 32]. A lot of the research focused on designing deep convolutional neural networks to improve accuracy [17]. Nevertheless, the development of a steady real-time Traffic Sign Recognition (TSR) still presents a challenging problem based on the latency in its testing time, which is crucial in making decisions based on the environment and real-world variability. TSR is one of the most well-known and widely discussed by lots of researchers. The primary goal of our research focuses on Taiwan's prohibitory sign detection and recognition.

The motivation arises from the lack of database, dataset, or research system for Taiwan's traffic sign recognition. Recognition of traffic sign contains two steps: localization and subsequent classification [33]. Due to the recent development of object detection, Fast RCNN [13] and Faster R-CNN [10, 42] provide predictions using hundreds of milliseconds to seconds. One of the most powerful and fastest algorithms based on deep neural networks is Yolo Darknet [38]. Yolo is about six times faster than Faster R-CNN [46]. The latest version of Yolo [41], Yolo V3 [39], has shown a significant advancement in real-time object detection, especially in the detection of smaller objects. Therefore, Yolo V3 is used for our object detection system.

Besides, the work adopts Spatial pyramid pooling (SPP) to pool the extracted features at variable scales and to get the superior features in max-pooling layers [15, 28]. SPP itself has been known as one of the most successful methods in computer vision [51], which has the flexibility of input scales and will improve the performance of Yolo V3.

The main contributions of the paper can be summarized as follows: (1) Since there is no pre-existing dataset for Taiwan's prohibitory sign, the system had to modify a database and collect the image by ourselves. Taiwan's prohibitory sign image dataset with 900 of total images was provided in this work. (2) Analyze the costs and benefits of using Yolo V3 and Yolo V3 SPP for Taiwan's prohibitory sign recognition. (3) Perform comparative analysis of Yolo V3 and Yolo V3 SPP with different weights provided by darknet framework (the best weight, the final weight, and the last weight). The observation and evaluation of specific models include essential metrics measurements, such as the mean average precision ( $m A P)$, workspace size, detection time, intersection over union (IoU), and the number of billion floating-point operations (BFLOPS).

The outline of the paper is as follows. Section 2 explains the related work of traffic sign detection and recognition systems. Section 3 briefly describes our proposed methodology. The dataset, the training, and the system's testing results will present in Section 4. Finally, conclusions and future work are proposed in Section 5.

\section{Related work}

\subsection{Traffic sign detection and recognition}

Recently, Faster R-CNN [53], You Only Look Once (Yolo) [37], and Single Shot Multi-Box Detector (SSD) [24] has become the most popular object detection frameworks. Yolo and SSD 
show the fast detection speed while struggling to precisely localize small objects because they both divide images into many grids that contain perhaps two or smaller objects. Faster R-CNN, the two-stage object detection framework, shows higher accuracy than Yolo and SSD [28]. The research related to a traffic sign, especially in Taiwan, could be seen on [51], where the primary goal of this work is to capture the traffic signs from the video footage through their proposed traffic signs capture software. Therefore, the Convolutional Neural Network (CNN) is used for the accuracy verification of the generated dataset. Base on C. Yanliang [50], the research combines the methods of the Adaboost classifier and Yolo V2 and tests the traffic signs. However, the results are a waste of time, and the accuracy is not good enough.

Typically, the researchers use free data collection to perform the experiment or capture the traffic sign picture by themselves. A variety of the various traffic sign databases are available, such as the GTSRB [44], the Chinese Traffic Sign Database, and the Tsinghua-Tencent $100 \mathrm{~K}$ (TT100K) [53]. Nevertheless, it is not easy to obtain a large number of high-quality image details. Different countries or states have their own traffic sign images. We focus on the prohibitory sign of Taiwan which consisted of no entry, no stopping, no parking, and speed limit images.

\subsection{The Darknet-53}

The Darknet-53 is a framework to train neural networks; it is open-source and is written in C and CUDA and serves as the basis for Yolo. The Darknet is fast and easy to install. It also supports CPU and GPU computation. The Darknet-53 is used as the framework for training Yolo, meaning it sets the architecture of the network [24, 37]. Darknet-53 Structure is shown in Fig. 1.

Darknet-53 is better than ResNet-101 and 1.5 times faster. It has a similar performance to ResNet-152, but it is two times faster. Darknet-53 also achieves the highest measured floatingpoint operations per second. The network structure utilizes the GPU to be better, and it makes processing time more efficient to evaluate and faster [40]. The weights of the custom detector are saved for every 100 until 1000 iterations, and it continues to be saved for every 10,000 iterations until it reaches the maximum batches. Recently, with the latest version of darknet while training, it provides the best weight, the final weight, and the last weight. Therefore, our work will do comparative weight analysis accordingly.

\subsection{Yolo V3}

Yolo V3 algorithm consisted of fully CNN and was proposed in 2018 [21, 48]. It is an algorithm for post-processing outputs from the neural network. Also, it divides the input image into grid cells of the same size and predicts bounding boxes and the probabilities for each grid cell [18]. Yolo V3 neural network consists of 106 layers and uses multi-scale fusion to make predictions. The input of the image size is $416 \times 416$ and combined with three scales [47]. Furthermore, the detection is done on the three separate layers [3]. The input dimensions width and height are $13 \times 13,26 \times 26$, and $52 \times 52$ [5]. Output tensors from those detection layers have the same widths and heights as their inputs, but depth is defined as for formula 1.

$$
\text { depth }=(4+1+\text { class probabilities }) \times 3
$$

where 4 is the number of bounding box properties such as width $\left(b_{w}\right)$, height $\left(b_{h}\right), x$ and $y$ position of the box $\left(b_{x}, b_{y}\right)$ inside the image; 1 is the probability that box contains the detectable object $(p c)$ and class probabilities for each of the classes $\left(c_{1}, c_{2}, \ldots, c_{5}\right)$. That sum 
Type

Filters Size

Output

$\begin{array}{llll}\text { Convolutional } & 32 & 3 \times 3 & 256 \times 256\end{array}$

Convolutional $64 \quad 3 \times 3 / 2 \quad 128 \times 128$

Convolutional $32 \quad 1 \times 1$

$1 \times$ Convolutional $64 \quad 3 \times 3$

Residual

$128 \times 128$

Convolutional $128 \quad 3 \times 3 / 2 \quad 64 \times 64$

Convolutional $64 \quad 1 \times 1$

$2 \times$ Convolutional $128 \quad 3 \times 3$

Residual

$64 \times 64$

Convolutional $256 \quad 3 \times 3 / 2 \quad 32 \times 32$

Convolutional $128 \quad 1 \times 1$

$8 \times$ Convolutional $256 \quad 3 \times 3$

Residual

$32 \times 32$

Convolutional $512 \quad 3 \times 3 / 2 \quad 16 \times 16$

$8 \times$\begin{tabular}{|lllc|}
\hline $\begin{array}{l}\text { Convolutional } \\
\text { Convolutional }\end{array}$ & 512 & $1 \times 1$ & \\
Cons & & \\
Residual & & & $16 \times 16$ \\
\hline Convolutional & 1024 & $3 \times 3 / 2$ & $8 \times 8$ \\
\hline
\end{tabular}

$4 \times$\begin{tabular}{|lccc|}
\hline $\begin{array}{l}\text { Convolutional } \\
\text { Convolutional }\end{array}$ & 512 & $1 \times 1$ & \\
Residual & & $3 \times 3$ & \\
Residual & & $8 \times 8$ \\
\hline
\end{tabular}

Avgpool Global

Connected $\quad 1000$

Softmax

Fig. 1 The Darknet-53 structure

is multiplied by three because each of the cells inside the grid can predict three bounding boxes. The backbone of Yolo V3 is Darknet-53 is shown in Fig. 1. It was made of 53 convolutional layers to capture deep features and has been proved to be more effective than Darknet-19, ResNet-101, or ResNet-152 [21]. The algorithm of Yolo V3 divides the input 
image into $S \times S$ grids. If the center point of the object's ground truth falls within a certain grid, the grid is responsible for detecting the object. Figure 2 shows the bounding boxes with dimension prior and location prediction. In Fig. $2, b_{x}, b_{y}, b_{w}, b_{h}$ are the $x, y$ center coordinates, width, and height of our prediction. $t_{x}, t_{y}, t_{w}$, and $t_{k}$ are the network outputs. $c_{x}$ and $c_{y}$ are the top-left coordinates of the grid, whereas $p_{w}$ and $p_{h}$ are anchors dimensions for the box [38, 40].

The loss function of parameter training of Yolo V3 is indicated as follows $[4,11]$ :

$$
L\left(s_{n}\right)=\left\{\begin{array}{c}
-\log \left(s_{n}\right) g_{n}=1 \\
-\log \left(1-s_{n}\right) g_{n}=0
\end{array}\right.
$$

where $n$ indicates the sample index; $s_{n} \in[0,1]$ represents the objectness score predicted by the network; the loss value measures the predicted probability that the $n$-th sample is an electrical component. Moreover, $g_{n}$ indicates the ground truth. It should be noted that $g_{n} \in[0,1]$ means if the $n$-th sample belongs to the class of objects.

\subsection{Spatial pyramid pooling (SPP)}

Spatial pyramid pooling (SPP) in terms of object recognition has been tremendously successful. Due to its simplicity, it is competitive with methods using more complex spatial models $[12,30]$. In the representation of the spatial pyramid, the input image is separated into a series of increasing finer grids at each pyramid phase. Furthermore, the output of the last convolutional feature maps is divided into proportional proportions into space containers in the SPP layer. The number of containers is fixed irrespective of the image size. In each space bin, the network adopts the max-pooling strategy [19, 54].

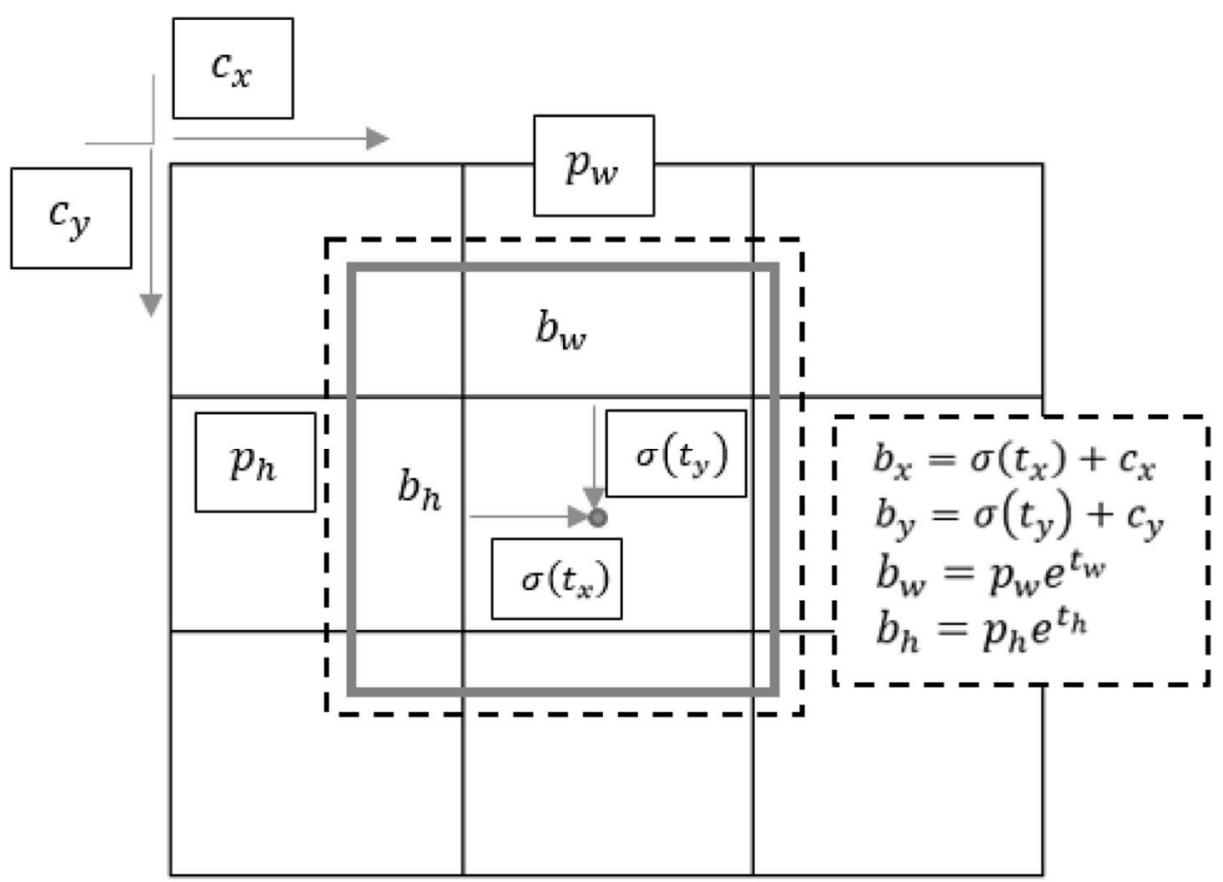

Fig. 2 Bounding boxes with dimension priors and location prediction 
SPP has some advantages as follows [16]: SPP not only allows us to produce images of predetermined proportions for processing but also allows us to feed images of varying measurements or weights during training. Next, training with variable-size images grows scale-invariance and reduces over-fitting. Moreover, SPP can pool features obtained at variable scales to the flexibility of input systems. A network structure with a spatial pyramid pooling (SPP) is shown in Fig. 3. In our experiment, SPP entered in the Yolo V3 configuration file.

\section{Proposed methodology}

vTaiwan's prohibitory sign detection and recognition are performed by the Yolo V3 and Yolo V3 SPP framework in one phase. BBox label tool is used for generating a bounding box for each sign. The labeling process is performed for four class labels (P1, P2, P3, and P4). Further, one image can host more than one bounding box. In the detection stage, a single class detector model was used and one class label belongs to one training model. The return values of the bounding box labeling tool are object coordinates in the form $\left(x_{1}, y_{1}, x_{2}, y_{2}\right)$. The Yolo input value is not in the form of object coordinates. Instead, the Yolo input value is the center point of the object and its width and height $(x, y, w, h)$. Hence, the system needs to change the bounding box coordinate into the Yolo input format. Equations (3)-(8) shows the transformation process $[1,11]$.

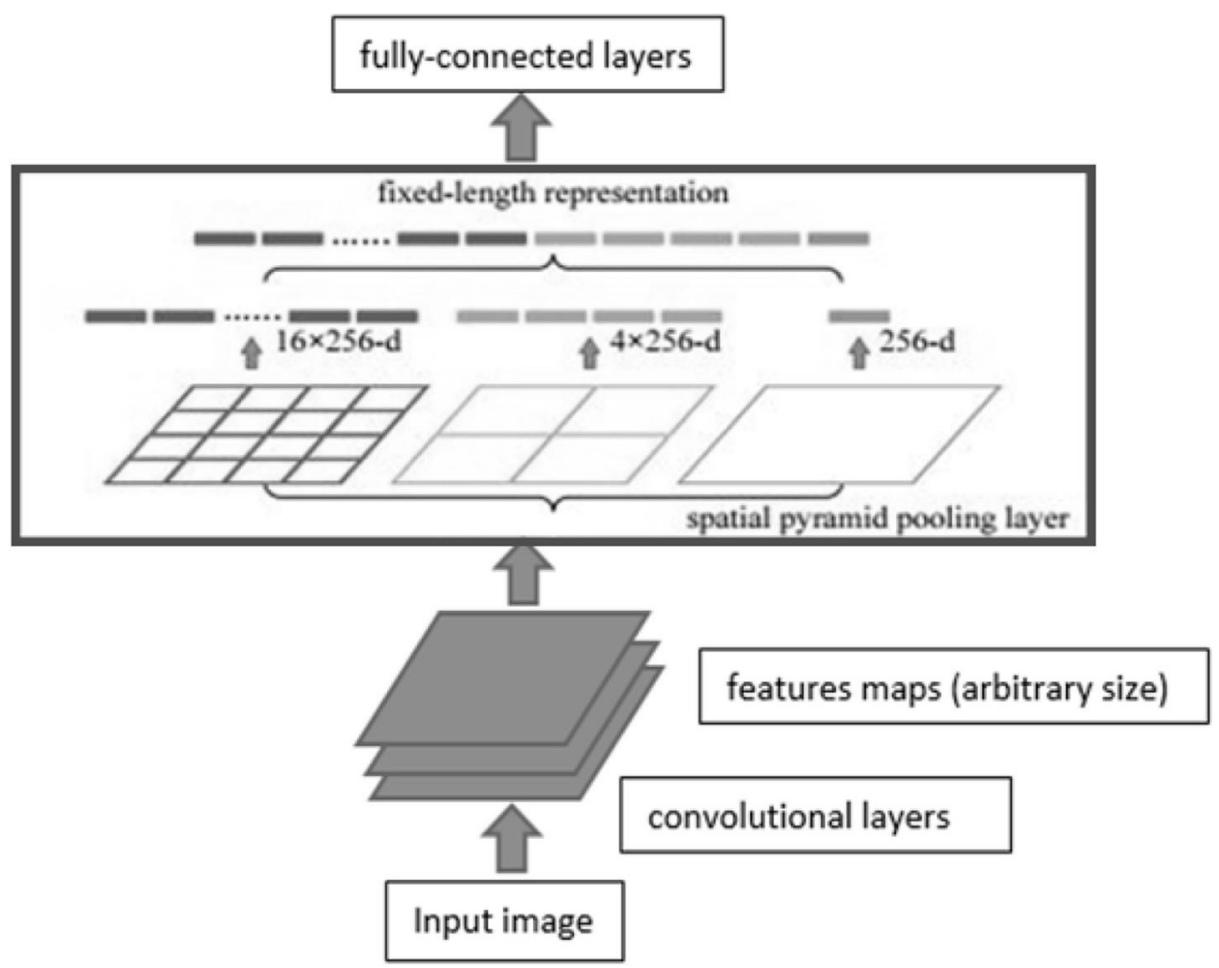

Fig. 3 Spatial Pyramid Pooling (SPP) layer 


$$
\begin{gathered}
d w=1 / W \\
x=\frac{\left(x_{1}+x_{2}\right)}{2} \times d w \\
d h=1 / H \\
y=\frac{\left(y_{1}+y_{2}\right)}{2} \times d h \\
w=\left(x_{2}-x_{1}\right) \times d w \\
h=\left(y_{2}-y_{1}\right) \times d h
\end{gathered}
$$

Where $W$ is the width of the image, $d w$ is the absolute width of the image, $H$ is the height of the image, and $d h$ is the absolute height of the image. In addition, float values relative to width and height of image $(d w, d h)$, it can be equal from 0.0 to 1.0 .

The purposed methodology is to recognize Taiwan's prohibitory sign using Yolo V3 based on spatial pyramid polling. Therefore, Fig. 4 describes our architecture of Yolo V3 SPP and Algorithm 1 describes object recognition with Yolo V3 SPP.

The Intersection over Union (IoU) is an overlap metric that indicates the degree of overlap between the model-generated target window and the original labeled frame [26]. It is not susceptible to scale and non-negative changes and is a common evaluation standard in target detection, also referred to as detection accuracy. The value of $I o U$ is the relation between the
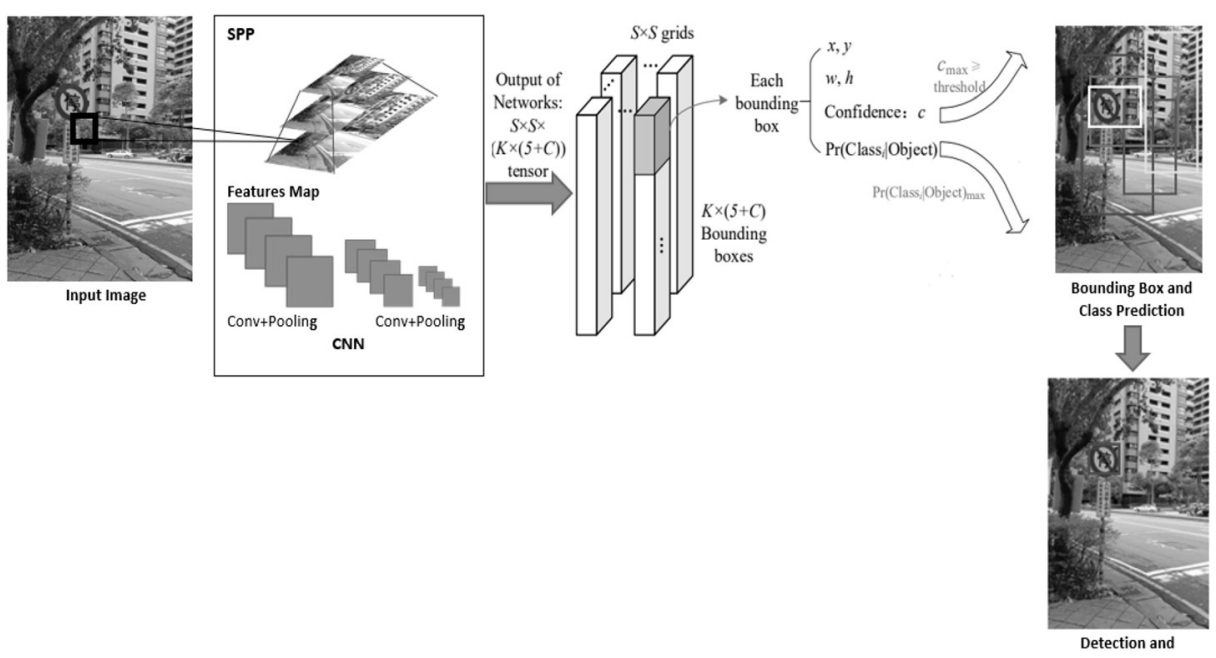

Fig. 4 The architecture of YOLO V3 SPP 
result of the detection, the reality of the ground truth, and the relationship between them [20]. $I o U$ calculates the overlap ratio between the boundary box of the prediction (pred) and groundtruth $(g t)$ shown in Eq. (9).

$$
I o U=\frac{\text { Area }_{\text {pred }} \cap \text { Area }_{g t}}{\text { Area }_{\text {pred }} \cup \text { Area }}
$$

Algorithm 1. Object detection process using Yolo V3 SPP

1. Split the input image into $\mathrm{S} \times \mathrm{S}$ grids, so every grid produces $K$ bounding boxes according to the calculation of the anchor boxes.

2. Use the $\mathrm{CNN}$ to extract all object features from the image and predict the $\boldsymbol{b}=\left[b_{x}, b_{y}, b_{w}, b_{h}, b_{c}\right]^{T}$ and the class $=[\text { class } P 1 \text {, class } P 2 \text {, class } P 3, \text { class } P 4]^{T}$.

3. Compare the maximum confidence $I o U_{\text {pred }}^{\text {truth }}$ of the $K$ bounding boxes with the threshold IoU thres.

4. If $I o U_{\text {pred }}^{\text {truth }}>I o U_{\text {thres }}$ means that the bounding box contains the object. Otherwise, the bounding box does not contain the object.

5. Choose the group of objects with the highest expected probability.

6. Finally, implement Non-Maximum Suppression (NMS) to conduct a maximum local search to remove duplicate boxes and outputs, then present object detection results.

The input of the target detection process using the Yolo V3 SPP algorithm is prohibitory sign Class P1, P2, P3, and P4. After the algorithm processes, the detected targets are bounded by bounding boxes, and the image class objects are associated. Also, the same target is given the same mark in each image and a uniform label is given to the same target. NMS is used for full local search to delete redundant boxes and output and to display object detection results. In our work, Yolo V3 uses down sampling in convolutional layers with a spatial model to receive important characteristics in max-pooling layers. For each picture, it applies three different sizes of the max pool with [route]. Through [route] uses various layers $-2,-4$ and $-1,-3,-5,-6$ in conv $_{5}$.

The comparison of Yolo V3 and Yolo V3 SPP with different weights provided by the Darknet framework in this work. The fully connected layers used for SoftMax classification and bounding-box regression are initialized from zero-mean Gaussian distributions with standard deviations 0.01 and 0.001 , respectively [13]. To speed up the training process and prevent over-fitting, the global learning rate is 0.001 , momentum is 0.9 , and parameter decay is 0.0005 . The learning rate hyperparameter controls the rate or speed at which the model learns. Specifically, it regulates the amount of apportioned error of the weights. The weights of the model are updated regularly based on the batch of training examples.

Our work arranges 6 model experiments shown in Table 1. Scale $=0.1,0.1$ was used for each Yolo V3, and Yolo V3 SPP. Hence, for an $n$-classes object detector, it is advisable to run the training for at least $2000 \times n$ batches. In our case, with four classes, so the maximum

Table 1 Experiment models

\begin{tabular}{llll}
\hline NO & Name & Model & Weight \\
\hline 1 & Yolo A & Yolo V3 & TSData_best.weights \\
2 & Yolo B & Yolo V3 & TSData_final.weights \\
3 & Yolo C & Yolo V3 & TSData_last.weights \\
4 & Yolo D & Yolo V3 SPP & Yolov3spp1_best.weights \\
5 & Yolo E & Yolo V3 SPP & Yolov3spp1_final.weights \\
6 & Yolo F & Yolo V3 SPP & Yolov3spp1_last.weights \\
\hline
\end{tabular}


batches number was 8000 . Moreover, the training will be processed for 8000 iterations. The darknet framework will automatically provide three different weights such as the best weight, the last weight, and the final weight if the training process reaches the maximum batches or iteration. While training darknet will recalculate the $m A P$ and resave the weight every 1000 iteration. During the training process, the weight that has the highest $m A P$ will save as the best weight. If the training process does not reach the maximum batches it will just obtain the best weight and the last weight.

\section{Experiment result and discussion}

The data set was split into $70 \%$ for training and $30 \%$ for testing. Since Taiwan's prohibitory signs are not exists, the system has had to configure the database and collect the image itself. In addition, our experiments focus on the prohibitive sign of Taiwan consisting of no entry images (235), no stopping images (250), speed limit images (185), and no parking images (230). In addition, a total of 900 images are used as shown in Table 2.

\subsection{Training result}

In the training process stage, data augmentation techniques such as cropping, padding, and horizontal flipping are applied. Such methods are used for the preparation of large neural networks. The experiment therefore conducts such operations during data augmentation with multiple settings like rotation range $=20$, zoom range $=0.10$, width shift range $=0.2$, height shift range $=0.2$, and shear range $=0.15$. Furthermore, a bounding box labelling tool [3] was used to manually detect and recognize traffic signs for the object to be detected. The results of the tools together with the class label are four points of the position coordinate. Then, before practicing, use the Yolo Annotation tool [4] to convert the label into Yolo format. The tool changes the values to a format which the training algorithm Yolo V3 can read. Additionally, the training environment is equipped with a Nvidia RTX1080Ti GPU accelerator11 GB memory, i7 CPU, and 16 GBDDR2 memory.

Figure 5 describes the training process using Yolo A (a) and Yolo B (b). The training configuration file uses network size $w i d t h=416$, and height $=416$ means that every image will be resized to the network size during training and detection. The training will be processed for 8000 iterations (max_batches), policy $=$ steps, and steps $=6400,7200$. The learning rate is a hyper-parameter that controls how to adjust the weights of the network. At the beginning of

Table 2 Taiwan's prohibitory signs class P1, P2, P3, and P4

\begin{tabular}{lllll}
\hline NO & Class & Sign & Total Image & Sign \\
\hline 1 & P1 & No entry & 235 \\
2 & P2 & No stopping & 250 & 230 \\
3 & P3 & No parking & 185 & 900 \\
4 & P4 & Speed Limit & Total & \\
\hline
\end{tabular}



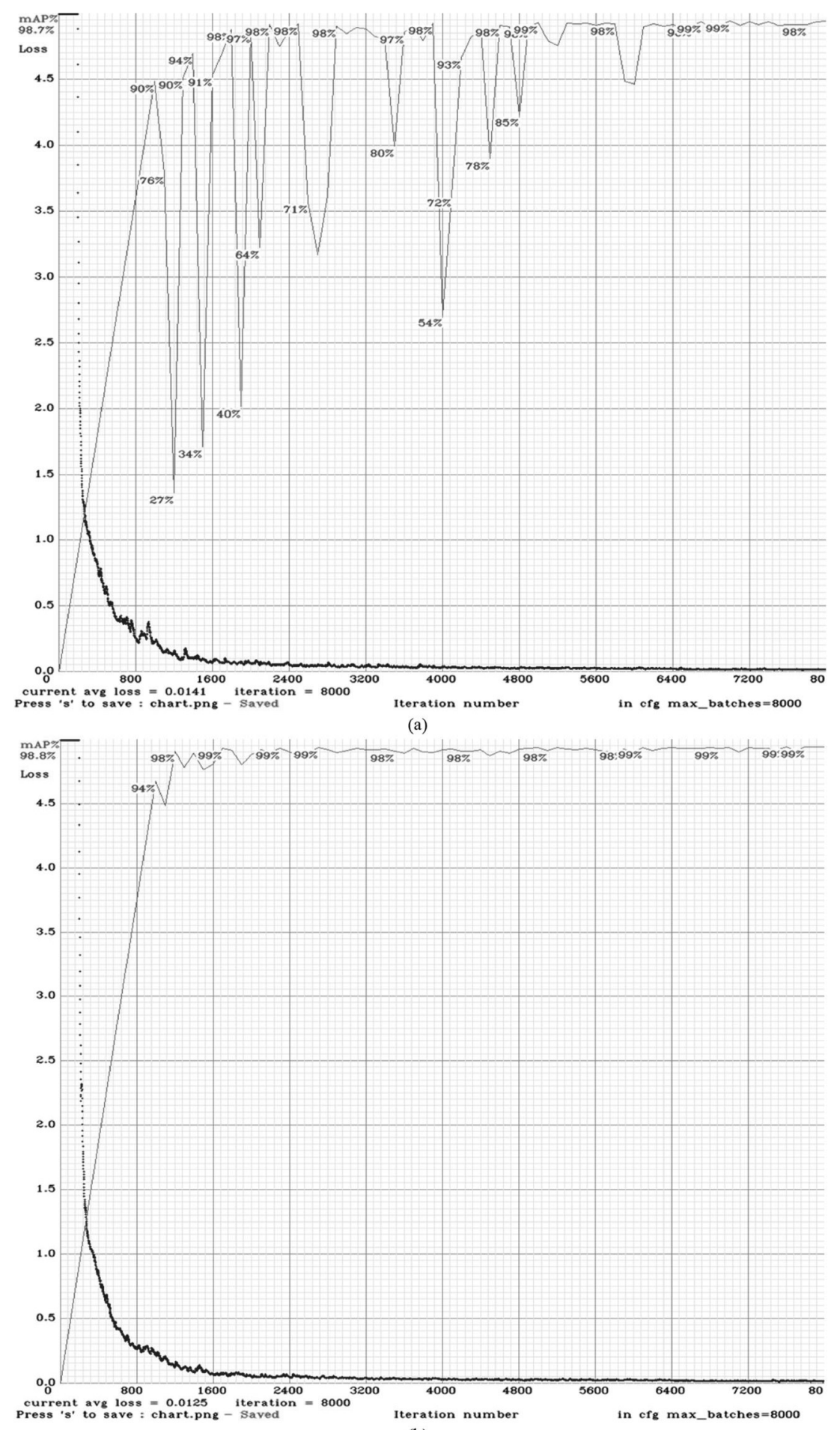

current ave loss $=0.00125$
Press ' $s$ ' to save : ohart.pne - Saved

(b)

Fig. 5 Training loss value, $m A P$, and $A P$ result using Yolo A (a) and Yolo C (b) 
the training process, the learning rate needs to be high. Typically, when one sets their learning rate and trains the model, one would only wait for the learning rate to decrease over time and for the model to converge eventually. Further, in the configuration file, the degradation in the learning rate is accomplished by first specifying that our learning rate decreasing policy is steps. In the above example, the learning rate will start from 0.001 and remain steady for 6400 iterations, and then multiply by scales to get the new learning rate. The summary of Fig. $5 \mathrm{~b}$ is that Yolo V3 SPP is more constant than Yolo V3 in Fig. 5a during the training process. Table 3 describes the detail of training loss value, AP, TP, FP, precision, recall, FI-score, Intersection over Union (IoU), and $m A P$ performance for all classes.

Table 3 shows the training loss value, $m A P, A P$, precision, recall, $\mathrm{F} 1$, and $I o U$ performance for all classes after training 8000 iterations. The average of the training validation loss for all classes is around 0.0133. Thus, our training model detected the objects with high accuracy. The training model converged after 7200 iterations and remained stable during the training phase. The loss value for Yolo V3 SPP is 0.0141, and for Yolo V3 is 0.0125 . Also, Table 3 shows that Yolo V3 SPP obtains maximum average $m A P$ 99.043\%. During $m A P$ calculation, Yolo V3 SPP loaded 107 layers, with total BFLOPS 65.312, while Yolo V3 loaded 114 layers with total BFLOPS 65.69. Furthermore, SPP could improve the total BFLOFS 0.378, thus make Yolo V3 SPP more accurate and constant. The average mean average precision $(m A P)$ is the integral over the precision $p(o)$.

$$
m A P=\int_{0}^{1} p(0) d o
$$

where $p(o)$ is the precision of the object detection. Precision and recall are represented by $[7,8,52]$ in Eqs. (11)-(12).

$$
\begin{gathered}
\text { Precision }=\frac{T P}{T P+F P}=T P / N \\
\text { Recall }=\frac{T P}{T P+F N}
\end{gathered}
$$

Where TP represents true positives, $\mathrm{FP}$ false positives, $\mathrm{FN}$ false negative, and $\mathrm{N}$ is the total number of objects retrieved (TP + FP). Another evaluation index, F1 [22] is shown as follows.

$$
F 1=\frac{2 \times \text { Precision } \times \text { Recall }}{\text { Precision }+ \text { Recall }}
$$

\subsection{Testing accuracy class P1, P2, P3, and P4}

Table 4 shows the testing accuracy for all classes (P1, P2, P3, and P4) of Taiwan's prohibitory sign. Class P2 got the maximum average accuracy around 97\%, followed by class P4 for $96.52 \%$, class P1 reached $96.54 \%$, and the minimum accuracy was $95.70 \%$ in class P3. Yolo V3 SPP scored the maximum accuracy compare to Yolo V3. Yolo D presented the highest accuracy, around $99.42 \%$, followed by Yolo E and Yolo $\mathrm{F}$ at $98.99 \%$. Finally, from these results, the conclusion is the best weight achieved the highest accuracy, the last weight and final weight got the same accuracy for both Yolo V3 and Yolo V3 SPP. 


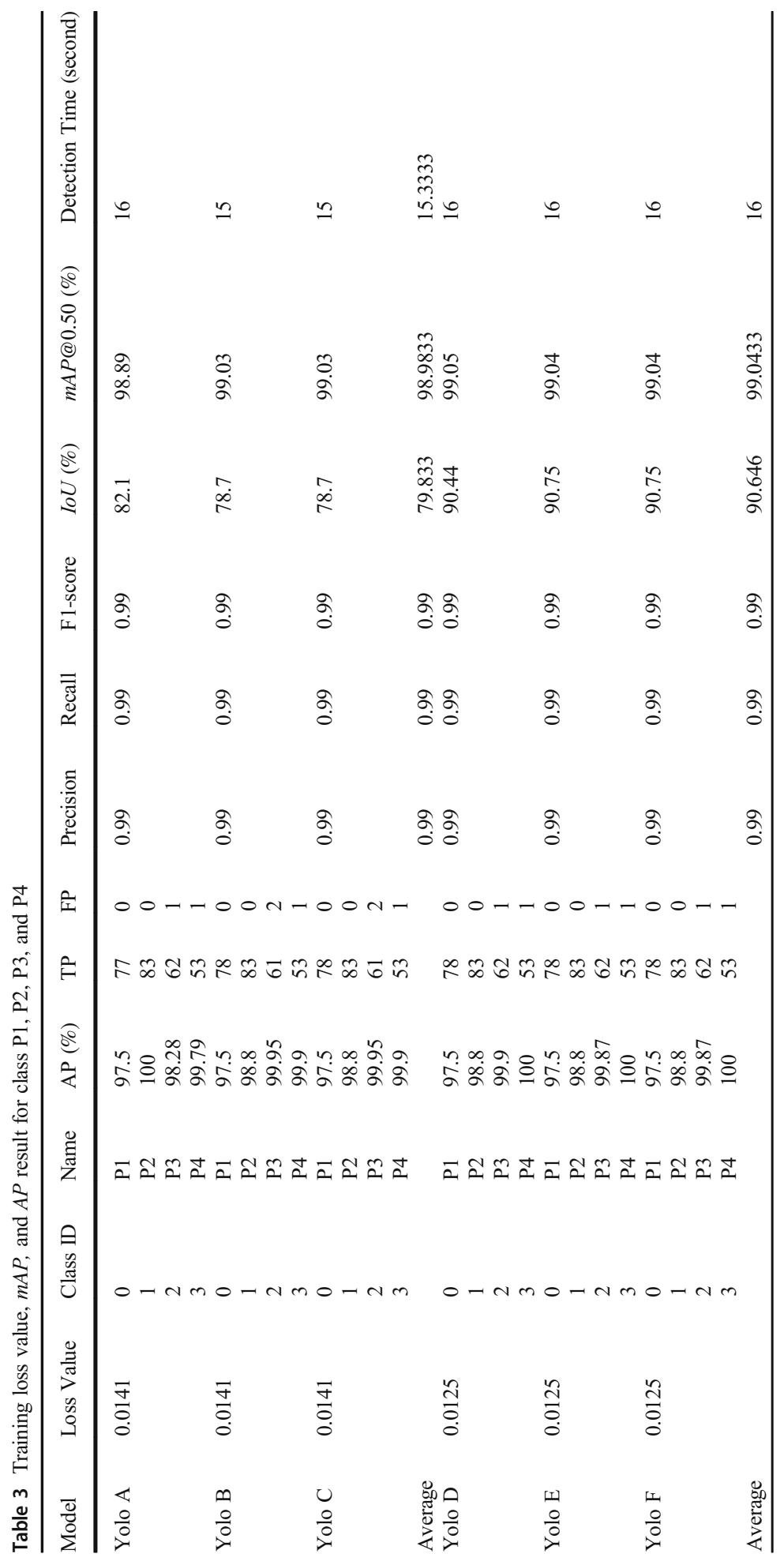


Table 4 Testing accuracy for class P1, P2, P3, and P4

\begin{tabular}{|c|c|c|c|c|c|}
\hline \multirow[t]{2}{*}{ Model } & \multicolumn{5}{|c|}{ Testing Accuracy } \\
\hline & $\mathrm{P} 1$ & $\mathrm{P} 2$ & P3 & $\mathrm{P} 4$ & Average \\
\hline Yolo A & 0.9346 & 0.9756 & 0.9402 & 0.9378 & 0.9470 \\
\hline Yolo B & 0.9298 & 0.9228 & 0.9053 & 0.9719 & 0.9324 \\
\hline Yolo C & 0.9298 & 0.9228 & 0.9053 & 0.9719 & 0.9324 \\
\hline Yolo D & 0.9985 & 0.9999 & 0.9971 & 0.9814 & 0.9942 \\
\hline Yolo E & 0.9985 & 0.9999 & 0.9971 & 0.9641 & 0.9899 \\
\hline Yolo F & 0.9985 & 0.9999 & 0.9971 & 0.9641 & 0.9899 \\
\hline Average & 0.9649 & 0.9702 & 0.9570 & 0.9652 & \\
\hline
\end{tabular}

\subsection{Experimental results and discussion}

We discuss twenty prohibitory sign images with several sizes and testing conditions. Table 5 presents the accuracy and time measurements of the experiments. Nevertheless, Yolo V3 SPP is generally more accurate for the different weights than Yolo V3. Further, Yolo D took the highest average accuracy at 99.0\%, followed by Yolo E and Yolo F 98.9\%. The accuracy of Yolo V3 SPP is increasing within the detection time, but it requires more time to detect the sign. For instance, the average time of detection was $0.516 \mathrm{~s}$ for Yolo D, while Yolo A required $0.508 \mathrm{~s}$. Moreover, Yolo B and Yolo C got the same accuracy of $92.3 \%$ while Yolo E and Yolo F receives $98.9 \%$.

Table 5 Recognition result using all models with various images and sizes

\begin{tabular}{|c|c|c|c|c|c|c|c|c|c|c|c|c|}
\hline \multirow[t]{2}{*}{ Image } & \multicolumn{2}{|c|}{ Yolo A } & \multicolumn{2}{|c|}{ Yolo B } & \multicolumn{2}{|c|}{ Yolo C } & \multicolumn{2}{|c|}{ Yolo D } & \multicolumn{2}{|c|}{ Yolo E } & \multicolumn{2}{|c|}{ Yolo F } \\
\hline & $\begin{array}{l}\text { Acc } \\
(\%)\end{array}$ & $\begin{array}{l}\text { Time } \\
\text { (s) }\end{array}$ & $\begin{array}{l}\text { Acc } \\
(\%)\end{array}$ & $\begin{array}{l}\text { Time } \\
\text { (s) }\end{array}$ & $\begin{array}{l}\text { Acc } \\
(\%)\end{array}$ & $\begin{array}{l}\text { Time } \\
\text { (s) }\end{array}$ & $\begin{array}{l}\text { Acc } \\
(\%)\end{array}$ & $\begin{array}{l}\text { Time } \\
\text { (s) }\end{array}$ & $\begin{array}{l}\text { Acc } \\
(\%)\end{array}$ & $\begin{array}{l}\text { Time } \\
\text { (s) }\end{array}$ & $\begin{array}{l}\text { Acc } \\
(\%)\end{array}$ & $\begin{array}{l}\text { Time } \\
\text { (s) }\end{array}$ \\
\hline P1-1.jpg & 0.966 & 0.523 & 0.980 & 0.514 & & & 0.972 & & 0.969 & 0.519 & 0.969 & 0.510 \\
\hline P1-2.jpg & 0.898 & 0.503 & 0.909 & 0.495 & 0.909 & 0.493 & 0.972 & & 0.965 & 0.511 & 0.965 & 0.503 \\
\hline P1-3.jpg & 0.837 & 0.526 & 0.891 & 0.513 & 0.891 & 0.499 & 0.993 & 0.525 & 0.993 & 0.520 & 0.993 & 0.513 \\
\hline P1-4.jpg & 0.949 & 0.518 & 0.975 & 0.523 & 0.975 & 0.510 & 0.992 & 0.528 & 0.991 & 0.511 & 0.991 & 0.506 \\
\hline P1-5.jpg & 0.867 & 0.510 & 0.895 & 0.495 & 0.895 & 0.499 & 0.990 & 0.515 & 0.991 & 0.507 & 0.991 & 0.517 \\
\hline P2-1.jpg & 0.976 & 0.504 & 0.983 & 0.503 & 0.983 & 0.513 & 0.988 & 0.506 & 0.987 & 0.516 & 0.987 & 0.524 \\
\hline P2-2.jpg & 0.965 & 0.506 & 0.919 & 0.510 & 0.919 & 0.510 & 0.997 & 0.512 & 0.997 & 0.505 & 0.997 & 0.509 \\
\hline P2-3.jpg & 1.000 & 0.499 & 1.000 & 0.521 & 1.000 & 0.509 & 0.999 & 0.528 & 0.999 & 0.524 & 99 & 0.519 \\
\hline P2-4.jpg & 0.883 & 0.515 & 0.888 & 0.518 & 0.888 & 0.516 & 0.987 & 0.513 & 0.987 & 0.530 & 0.987 & 0.507 \\
\hline P2-5.jpg & 0.906 & & & & & & & & & & & \\
\hline P3-1.jpg & 0.971 & 0.517 & 0.983 & 0.506 & 0.983 & 0.521 & 0.993 & 0.509 & 0.991 & 0.540 & 0.991 & 0.522 \\
\hline P3-2.jpg & 0.823 & 0.514 & 0.874 & 0.508 & 0.874 & 0.504 & 0.992 & 0.515 & 0.992 & 0.514 & 0.992 & 0.513 \\
\hline P3-3.jpg & 0.997 & 0.513 & 0.997 & 0.500 & 0.997 & 0.497 & 0.999 & 0.512 & & & & 0.523 \\
\hline P3-4.jpg & 0.995 & 0.506 & 0.997 & 0.516 & 0.997 & 0.501 & 0.999 & 0.514 & 0.999 & 0.511 & 0.999 & 0.520 \\
\hline P3-5.jpg & 0.935 & 0.497 & 0.893 & 0.499 & 0.893 & 0.525 & 0.979 & 0.523 & 0.975 & 0.527 & 0.975 & 0.505 \\
\hline P4-1.jpg & 0.879 & 0.487 & 0.861 & 0.509 & 0.861 & 0.509 & 0.992 & 0.502 & 0.992 & 0.517 & 0.992 & 0.512 \\
\hline P4-2.jpg & 0.988 & 0.490 & 0.989 & 0.509 & 0.989 & 0.507 & 0.997 & 0.500 & 0.996 & 0.527 & 0.996 & 0.505 \\
\hline $\mathrm{P} 4-3 . j p g$ & 0.953 & 0.497 & 0.894 & 0.494 & 0.894 & 0.506 & 0.998 & 0.525 & 0.998 & 0.512 & 0.998 & 0.517 \\
\hline P4 4.jpg & 0.891 & 0.518 & 0.751 & 0.513 & 0.751 & 0.506 & 0.994 & 0.525 & 0.994 & 0.510 & 0.994 & 0.510 \\
\hline P4-5.jpg & 0.897 & 0.518 & 0.842 & 0.518 & 0.842 & 0.504 & 0.987 & 0.520 & 0.986 & 0.506 & 0.986 & 0.508 \\
\hline Average & 0.929 & 0.508 & 0.923 & 0.508 & 0.923 & 0.507 & 0.990 & 0.516 & 0.989 & 0.517 & 0.989 & 0.513 \\
\hline
\end{tabular}


The experimental results indicate (1) Yolo V3 SPP is more robust than Yolo V3; (2) If the system wants to get the maximum accuracy, use the best weight. However, if we want to focus on detection time, the last weight is the fastest, among others. (3) Both of last weight and final weight will provide the same result of the accuracy, although they have different time detection. The last weight time detection is faster than the final weight.

Moreover, both convolution subsampling and max-pooling receive different advantages. Convolution subsampling can be better exchanged, apparently in the subsequent upsampling layers. Max pooling acts somewhat eliminating some high-frequency noises from the images by choosing only maximum values from the adjacent regions. By combining both, SPP seems to be leveraging the benefits of both, improving the backbone network of Yolo V3. Figure 6a-c gave the test results (P4-1.jpg) for the Yolo V3 model with an average accuracy of around $86.75 \%$ and a detection time of 0.5023 s. Further, Fig. 6d-f showed the test results for Yolo V3 SPP using the same image. The average accuracy was $99.21 \%$, and the detection time was $0.5109 \mathrm{~s}$. The system can detect and recognize Taiwan's prohibitory sign of class P4 very well. In Fig. 7a-c, Yolo V3 failed to detect all class P1's signs in the image, detecting only a single sign. However, Yolo V3 SPP was able to detect all signs well in Fig. 7d-f.

Yolo V4 [2] has recently been published and has been very successful compared to previous detectors and other known object detectors. Furthermore, Yolo V4 combines some features such as Weighted-Residual-Connections (WRC), Cross-Stage-Partial-connections (CSP), Cross Mini-Batch Normalization (CmBN), Self-adversarial-training (SAT) and Mishactivation, Mosaic data augmentation, Drop Block regularization, and $C I o U$ loss. Most of the
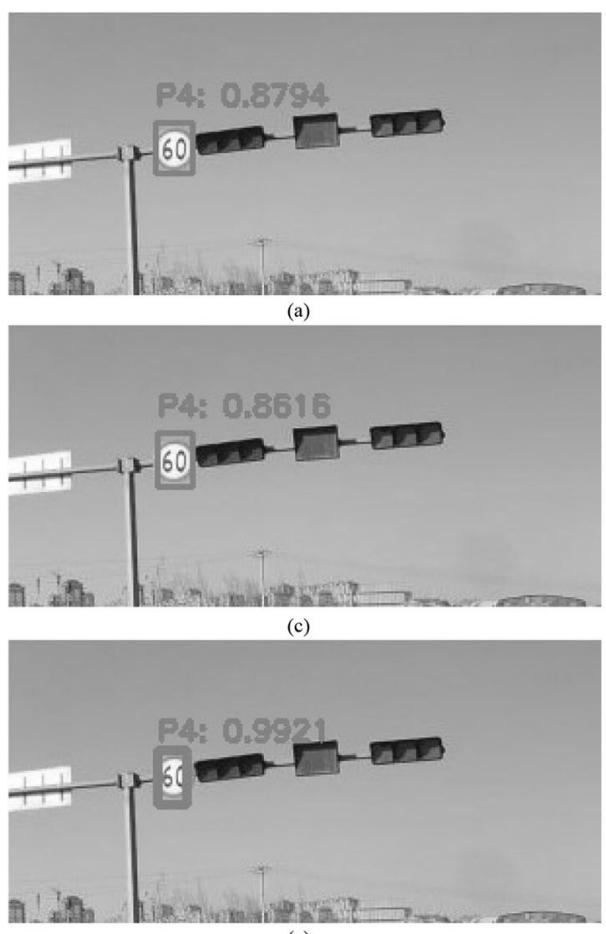

(e)

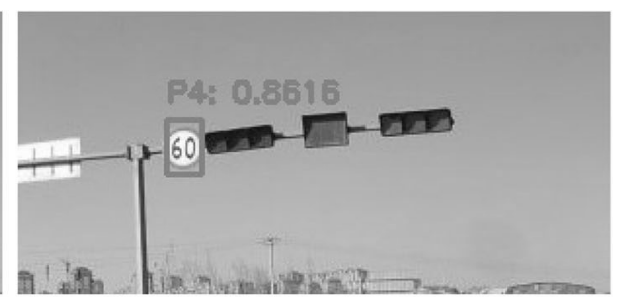

(b)
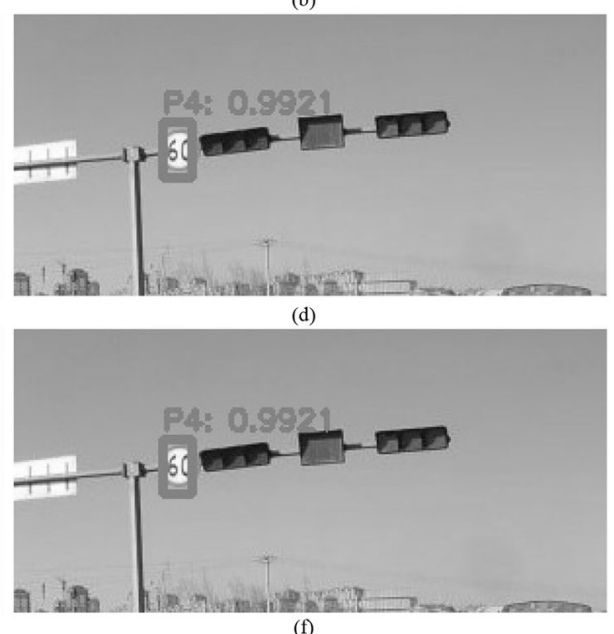

Fig. 6 Taiwan's prohibitory sign (Class P4) recognition result using Yolo A (a), Yolo B (b), Yolo C (c), Yolo D (d), Yolo E (e) and Yolo F (f) 

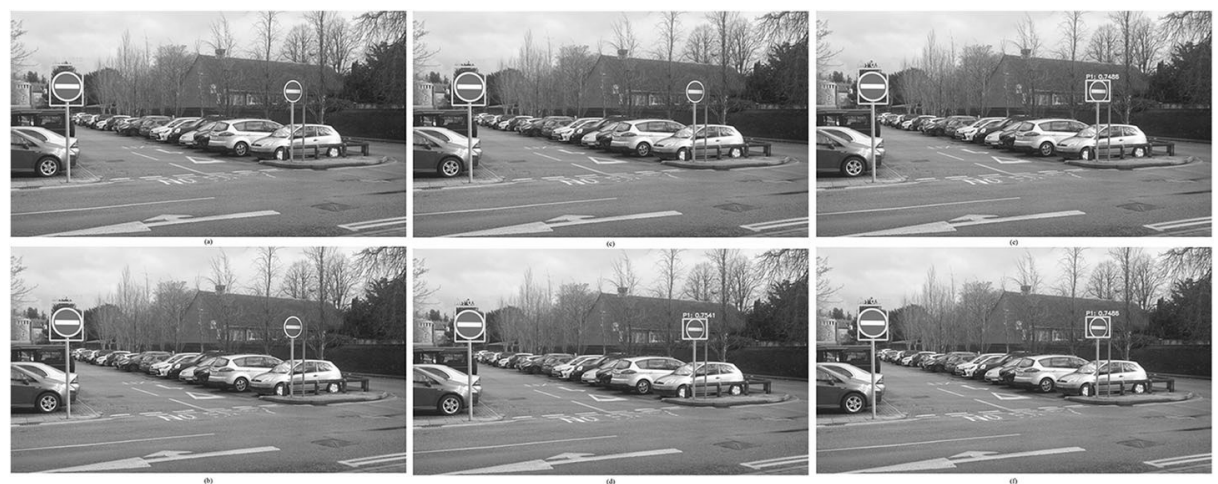

Fig. 7 Taiwan's prohibitory sign (Class P1) recognition result using Yolo A (a), Yolo B (b), Yolo C (c), Yolo D (d), Yolo E (e) and Yolo F (f)

precise modern models require several GPUs to work with a large mini-batch size, and this means that training with one GPU is very slow and unworkable. Yolo V4 solves this problem by making an object detector that can be trained for a smaller mini-batch size on a single GPU. Therefore, Yolo V4 has subsequent benefits as follows: (1) It is an effective and robust object detection model that allows anyone with a $1080 \mathrm{Ti}$ or $2080 \mathrm{Ti}$ GPU to train a super-fast and precise object detector. (2) The importance of state-of-the-art "Bag-of-Freebies" and "Bag-ofSpecials" object detection methods through detector training has been established. (2) The adjusted state-of-the-art methods, including CBN (Cross-iteration batch normalization), PAN (Path aggregation network), etc., are now more practical and fit for single GPU training. Perfect combination features from Yolo V4 can be employed as best-practice for future research studies and advancements.

\section{Conclusion}

In this paper, the Spatial Pyramid Pooling (SPP) was implemented in the Yolo V3 configuration file. SPP was applied to feed images with varying sizes or scales during training. The experimental results show that SPP can improve the effectiveness of recognizing Taiwan's prohibitory sign. $m A P$ comparison of all models shows that Yolo V3 SPP (Yolo D, Yolo E, and Yolo F) is outperforming Yolo V3 (Yolo A, Yolo B, and Yolo C). In addition, $m A P$ results show that the Yolo V3 SPP model gives the best performance compared to Yolo V3. Finally, based on all of the experiment results, the best weight will provide the highest accuracy. Hence, the final weight and last weight will produce the same accuracy. The fastest time detection is the last weight.

In future work, our plan is to extend the dataset focus not only in Taiwan's prohibitory signs but also all Taiwan's traffic signs. Our work also tries to expand the data set through the GAN network [6, 9, 49] and Contrast-Limited Adaptive Histogram Equalization (CLAHE) $[23,29]$ to achieve better results during training and testing. Also, in future research we will implement the newest Yolo V4 the Optimal Speed and Accuracy of Object Detection [2] and use other datasets as benchmarks such as TT100K dataset [53] and BTSD dataset [31, 45].

Acknowledgments This work is supported by the Ministry of Science and Technology, Taiwan. The Nos are MOST-107-2221-E-324 -018 -MY2 and MOST-106-2218-E-324 -002, Taiwan. This research is also partially sponsored by Chaoyang University of Technology (CYUT) and the Higher Education Sprout Project, Ministry of 
Education (MOE), Taiwan, under the project name: "The R\&D and the cultivation of talent for healthenhancement products."

\section{Compliance with ethical standards}

Conflict of interest The authors declared that they have no conflicts of interest in this work

\section{References}

1. (2019) Bbox label tool. In: https://github.com/puzzledqs/BBox-Label-Tool. https://github. com/puzzledqs/BBox-Label-Tool

2. Bochkovskiy A, Wang C-Y, Mark Liao H-Y (2020) YOLOv4: optimal speed and accuracy of object detection. arXiv:200410934

3. Chen Q, Liu L, Han R, et al (2019) Image identification method on high speed railway contact network based on YOLO v3 and SENet. In: Chinese Control Conference, CCC, pp 8772-8777

4. Chen H, He Z, Shi B, Zhong T (2019) Research on recognition method of electrical components based on YOLO V3. IEEE Access. 7:157818-157829. https://doi.org/10.1109/ACCESS.2019.2950053

5. Corovic A, Ilic V, Duric S, et al (2018) The real-time detection of traffic participants using YOLO algorithm. In: 2018 26th telecommunications forum, TELFOR 2018 - proceedings

6. Creswell A, White T, Dumoulin V, et al (2017) Generative adversarial networks: an overview. CoRR abs/ 1710.0:1-14

7. Dewi C, Chen R-C (2019) Random Forest and support vector machine on features selection for regression analysis. International journal of innovative computing. Information and Control (IJICIC) 15:2027-2038

8. Dewi C, Chen R-C (2019) Human activity recognition based on evolution of features selection and random Forest. In: 2019 IEEE international conference on systems, man and cybernetics (SMC), pp 2496-250

9. Dewi C, Chen R-C, Hendry, Liu Y-T (2019) Similar Music Instrument Detection via Deep Convolution YOLO-Generative Adversarial Network. In: 2019 IEEE 10th International Conference on Awareness Science and Technology (iCAST). pp 1-6

10. Dewi C, Chen RC, Hendry, Hung H Te (2020) Comparative Analysis of Restricted Boltzmann Machine Models for Image Classification. In: Lecture Notes in Computer Science (including subseries Lecture Notes in Artificial Intelligence and Lecture Notes in Bioinformatics), pp 285-296

11. Dewi C, Chen RC, Liu Y-T (2020) Taiwan Stop Sign Recognition with Customize Anchor. In: ICCMS '20, February 26-28, 2020, Brisbane, QLD, Australia

12. Dewi C, Chen R-C, Tai S-K (2020) Evaluation of robust spatial pyramid pooling based on convolutional neural network for traffic sign recognition system. Electronics 9:889

13. Girshick R (2015) Fast R-CNN. In: Proceedings of the IEEE International Conference on Computer Vision

14. Girshick R, Donahue J, Darrell T, Malik J (2014) Rich feature hierarchies for accurate object detection and semantic segmentation. In: Proceedings of the IEEE Computer Society Conference on Computer Vision and Pattern Recognition, pp 580-587

15. Grauman K, Darrell T (2005) The pyramid match kernel: discriminative classification with sets of image features. In: Proceedings of the IEEE International Conference on Computer Vision, pp 1458-1465

16. He K, Zhang X, Ren S, Sun J (2015) Spatial pyramid pooling in deep convolutional networks for visual recognition. IEEE Trans Pattern Anal Mach Intell 37:1-14. https://doi.org/10.1109/TPAMI.2015.238982

17. Hu Y, Wang FY, Liu X (2014) A CPSS approach for emergency evacuation in building fires. IEEE Intell Syst 29:1541-1672. https://doi.org/10.1109/MIS.2014.38

18. Hu Y, Wu X, Zheng G, Liu X (2019) Object detection of UAV for anti-UAV based on improved YOLO v3. In: 2019 Chinese control conference (CCC), ppp 8386-8390

19. Huang Z, Wang J (2019) DC-SPP-YOLO: dense connection and spatial pyramid pooling based YOLO for object detection. CoRR abs/1903.0:1-23

20. Huang Y-Q, Zheng J-C, Sun S-D, Yang CF, Liu J (2020) Optimized YOLOv3 algorithm and its application in traffic flow detections. Appl Sci 10. https://doi.org/10.3390/app10093079

21. Jost Tobias Springenberg Alexey Dosovitskiy, Brox T, Riedmiller MA (2014) Striving for Simplicity: The All Convolutional Net. CoRR abs/1412.6:1-14

22. Kang H, Chen C (2020) Fast implementation of real-time fruit detection in apple orchards using deep learning. Comput Electron Agric 168:105108. https://doi.org/10.1016/j.compag.2019.105108

23. Kim SJ, Min BS, Lim DK, Lee JH (2013) Determining parameters in contrast limited adaptive histogram equalization. The 7 th international conference on information security and assurance proceedings 
24. Liao X, Li K, Yin J (2017) Separable data hiding in encrypted image based on compressive sensing and discrete fourier transform. Multimed Tools Appl 76:20739-20753. https://doi.org/10.1007/s11042-016-3971-4

25. Liao X, Yin J, Guo S, Li X, Sangaiah AK (2018) Medical JPEG image steganography based on preserving inter-block dependencies. Comput Electr Eng 67:320-329. https://doi.org/10.1016/j. compeleceng.2017.08.020

26. Liu J, Huang Y, Peng J, Yao J, Wang L (2018) Fast object detection at constrained energy. IEEE Trans Emerg Top Comput 6:409-416. https://doi.org/10.1109/TETC.2016.2577538

27. Liu C, Li S, Chang F, Wang Y (2019) Machine vision based traffic sign detection methods: review, analyses and perspectives. IEEE Access 7:86578-86596. https://doi.org/10.1109/access.2019.2924947

28. Liu Z, Du J, Tian F, Wen J (2019) MR-CNN: a multi-scale region-based convolutional neural network for small traffic sign recognition. IEEE Access 7:57120-57128. https://doi.org/10.1109 /ACCESS.2019.2913882

29. Liu C, Sui X, Kuang X, Liu Y, Gu G, Chen Q (2019) Adaptive contrast enhancement for infrared images based on the neighborhood conditional histogram. Remote Sensing 11. https://doi.org/10.3390/rs11111381

30. Ma C, Miao Z, Li M (2015) Saliency weighted spatial pyramid representation for object recognition. In: IET Conference Publications

31. Mathias M, Timofte R, Benenson R, Van Gool L (2013) Traffic sign recognition - how far are we from the solution? In: Proceedings of the International Joint Conference on Neural Networks

32. Møgelmose A, Trivedi MM, Moeslund TB (2012) Vision-based traffic sign detection and analysis for intelligent driver assistance systems: perspectives and survey. IEEE Trans Intell Transp Syst 13:1484-1497. https://doi.org/10.1109/TITS.2012.2209421

33. Nikonorov A, Petrov M, Yakimov P (2013) Traffic sign detection on GPU using color shape regular expressions. In: Proceedings of the 4th international workshop on image mining. Theory and applications, IMTA 2013, In Conjunction with VISIGRAPP 2013

34. Ohn-Bar E, Trivedi MM (2015) Learning to detect vehicles by clustering appearance patterns. IEEE Trans Intell Transp Syst 16:2511-2521. https://doi.org/10.1109/TITS.2015.2409889

35. Philipsen MP, Jensen MB, Satzoda RK, Trivedi MM, Møgelmose A, Moeslund TB (2015) Day and nighttime drive analysis using stereo vision for naturalistic driving studies. In: IEEE Intelligent Vehicles Symposium, Proceedings. pp 1226-1231

36. Qian X, Feng H, Zhao G, Mei T (2014) Personalized recommendation combining user interest and social circle. IEEE Trans Knowl Data Eng 26:1763-1777. https://doi.org/10.1109/TKDE.2013.168

37. Redmon J Darknet: Open Source Neural Networks in C. http://pjreddie.com/darknet/

38. Redmon J, Farhadi A (2017) YOLO9000: better, faster, stronger. In: Proceedings - 30th IEEE conference on computer vision and pattern recognition, CVPR 2017, pp 6517-6525

39. Redmon J, Farhadi A (2018) YOLOv3: an incremental improvement. CoRR abs/1804.0:1-6

40. Redmon J, Farhadi A (2018) YOLOv3: An Incremental Improvement

41. Redmon J, Divvala S, Girshick R, Farhadi A (2016) You only look once: unified, real-time object detection. In: Proceedings of the IEEE Computer Society Conference on Computer Vision and Pattern Recognition

42. Ren S, He K, Girshick R, Sun J (2017) Faster R-CNN: towards real-time object detection with region proposal networks. IEEE Trans Pattern Anal Mach Intell 39:1137-1149. https://doi.org/10.1109 /TPAMI.2016.2577031

43. Ruta A, Li Y, Liu X (2010) Real-time traffic sign recognition from video by class-specific discriminative features. Pattern Recogn 43:416-430. https://doi.org/10.1016/j.patcog.2009.05.018

44. Stallkamp J, Schlipsing M, Salmen J, Igel C (2011) The German traffic sign recognition benchmark: a multiclass classification competition. In: Proceedings of the International Joint Conference on Neural Networks

45. Timofte R, Zimmermann K, Van Gool L (2009) Multi-view traffic sign detection, recognition, and 3D localisation. In: 2009 Workshop on applications of computer vision, WACV 2009

46. Wang CY, Cheng-Yue R (2017) Traffic sign detection using you only look once framework. Neurocomputing

47. Wu Y, Li Z, Chen Y, Nai K, Yuan J (2020) Real-time traffic sign detection and classification towards real traffic scene. Multimed Tools Appl 79:18201-18219

48. Xu H, Srivastava G (2020) Automatic recognition algorithm of traffic signs based on convolution neural network. Multimed Tools Appl 79:11551-11565

49. Yang J, Kannan A, Batra D, Parikh D (2019) LR-GAN: layered recursive generative adversarial networks for image generation. In: 5th international conference on learning representations, ICLR 2017 - conference track proceedings, pp 1-21

50. Yanliang C, Yongran L, Baisen W, et al (2019) Applying deep learning technology to image recognition of traffic signs. In: 2019 International conference on technologies and applications of artificial intelligence, pp 1-4

51. Yen-Zhang, Huang (2018) Building a traffic signs open dataset in Taiwan and verify it by convolutional neural network 
52. Yuan Y, Xiong Z, Wang Q (2017) An incremental framework for video-based traffic sign detection, tracking, and recognition. IEEE Trans Intell Transp Syst 18:1918-1929. https://doi.org/10.1109 /TITS.2016.2614548

53. Zhu Z, Liang D, Zhang S, et al (2016) Traffic-sign detection and classification in the wild. In: Proceedings of the IEEE Computer Society Conference on Computer Vision and Pattern Recognition, pp 2110-2118

54. Zhu R, Mao XJ, Zhu QH, et al (2016) Text detection based on convolutional neural networks with spatial pyramid pooling. In: Proceedings - International Conference on Image Processing, ICIP

Publisher's note Springer Nature remains neutral with regard to jurisdictional claims in published maps and institutional affiliations.

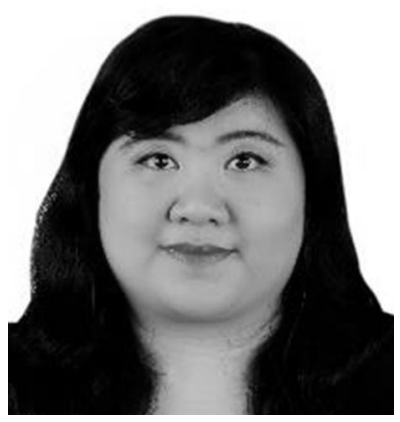

Christine Dewi received The B.S. Degree (S. Kom.) from the Informatics engineering study program in 2010, and a Master of Computer Science (M.Cs.) from the Master of Information Systems study program in 2012, both from the Faculty of Information Technology, Satya Wacana Christian University, Salatiga, Indonesia. Moreover, in 2018 she started her Ph.D. at the College of Informatics, Chaoyang University of Technology, Taiwan. Her current research interests include Image Processing, Computer Vision, Object Detection and Recognition, Artificial Intelligence, and Machine Learning.

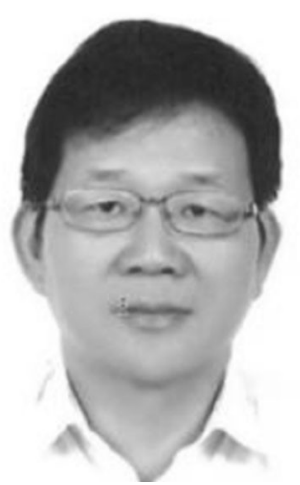

Rung-Ching Chen received a B.S. from the Department of Electrical Engineering in 1987, and an M. S. from the Institute of Computer Engineering in 1990, both from National Taiwan University of Science and Technology, Taipei, Taiwan. In 1998, he received his Ph.D. from the Department of Applied Mathematics in computer science, National Chung Hsing University. He is now a distinguished professor in the Department of Information Management, Taichung, Taiwan. His research interests include network technology, pattern recognition, and knowledge engineering, IoT and data analysis, and applications of Artificial Intelligence. 


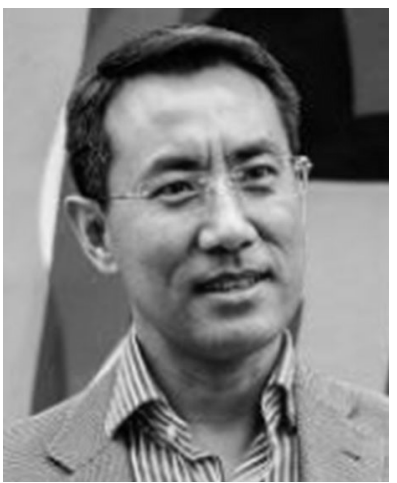

Hui Yu is a Professor at the CCI Faculty of the University of Portsmouth. His Ph.D. work won the Best Ph.D. Thesis Prize, EPSRC DHPA Awards and Vice-Chancellor Travel Prize, etc. He previously held a research appointment with the University of Glasgow. Moreover, his research interests include vision, computer graphics, and application of machine learning to these areas, particularly in research areas such as image/video processing and analysis, 3D/4D sensing, reconstruction and geometric processing, Human motion understanding, and effective analysis. 